\title{
Optimum Shape of High Speed Impactor for Concrete Targets Using PSOA Heuristic
}

\author{
Francesco Ragnedda, Mauro Serra \\ Department of Mathematics and Computer Science, University of Cagliari; \\ Department of Structural Engineering, University of Cagliari \\ E-mail:ragnedda@unica.it,serrama@unica.it \\ Received November 16, 2009; revised January 20, 2010; accepted February 4, 2010
}

\begin{abstract}
The present paper deals with the optimum shape design of an absolutely rigid impactor which penetrates into a semi-infinite concrete shield. The objective function to maximize is the depth of penetration (DOP for short) of the impactor; in the case of impactors with axisymmetric shapes DOP is calculated using formulas obtained by Ben-Dor et al. [1-3] with the method of local variations [4] and based on the mechanical model proposed by Forrestal and Tzou [5]. In the present paper we show that using a different class of admissible functions, more general than the axisymmetric one, better results can be obtained. To solve the formulated optimization problem we used a custom version of the particle swarm optimization method (briefly denoted by PSOA), a very recent numerical optimization algorithm of guided random global search. Numerical results show the optimal shape for various types of shields and corresponding DOP; some Ben-Dor et al. [1-3] results are compared to solutions obtained.
\end{abstract}

Keywords: Impactor; Optimization; Particle swarm; Global Search

\section{Introduction}

A problem important both for the civil world as well as for military research is to evaluate the depth of penetration (DOP) of a high-speed impactor when it penetrates a shield. A quite general solution to such problem was proposed by Forrestal et al. [5-7] in the case of concrete targets. Four different models for the shield material are considered: incompressible elastic-plastic (model 1), incompressible elastic-cracked-plastic (model 2), compressible elastic-plastic (model 3) and compressible elastic-cracked-plastic (model 4). Supposing the impactor's shape is axysimmetric with an unknown starting radius (flat nose), given length and final radius, (we call $K_{0}$ such class of shapes) and using the Forrestal et al. [5-7] model, Ben-Dor et al. $[1-3,8]$ we investigated the maximum depth of penetration and found corresponding numerical solutions. From a mathematical point of view, the problem is reduced to a non-classical variational one for a functional that is a function of integrals of the unknown impactor's shape; the technique used to solve it is that of local variations, proposed by Banichuk et al.[4]. In the following we shall define a new class of impactors and show how to get corresponding formulas.

It is worth noting that in the current literature, some penetration problems for three-dimensional bodies of optimal shape are also present [9-12] and very recently Banichuk and Ivanova [13] obtained new results applying the Forrestal and Tzou model to pyramidal impactors.

\section{Model building}

Let us consider an impactor whose impact velocity is normal to the concrete shield and has modulus $v_{i m p}$. The impactor has a shape built in the following manner: consider a body of revolution obtained revolving a curve of function $y=y(z)$ in the Cartesian coordinates (Oxy) around $z$ axis by $2 \pi$ and let $0 \leq z \leq L$, where $L$ is the length of the impactor; we suppose also that $y(z) \geq z L / R$ The function $y(z)$ has the unknown value $y(0)=y_{0}$ for $z=$ 0 and the fixed value $R$ for $z=\mathrm{L}$ (Figure 1). Next we cut such body with two planes passing through $y$ axis and crossing the $z x$ plane respectively through $(0,0),(L, R)$ and $(0,0),(L,-R)$. Excluding the two external parts of the body we get a new non-axisymmetric shape called 


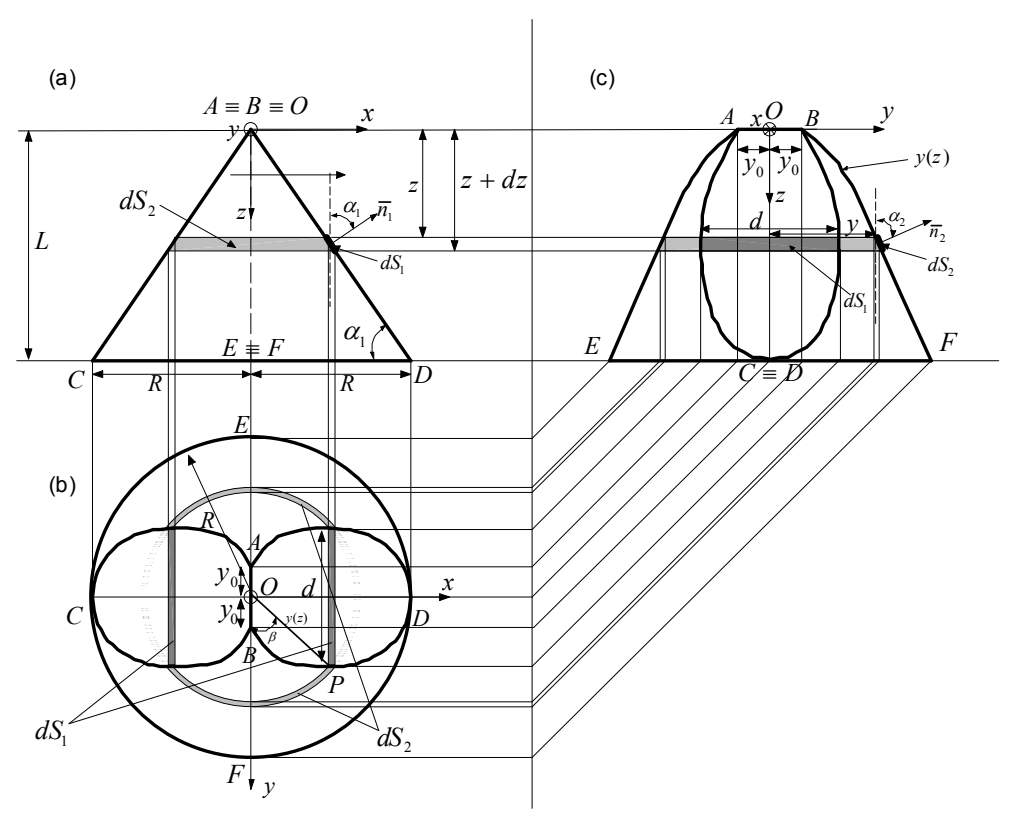

Figure 1. Screwdriver impactor.

"screwdriver shape" or shape of class $K_{1}$ (Figure 1). In the shape so obtained we can distinguish two distinct surfaces: $S_{1}$, corresponding to the plane part, and $S_{2}$ corresponding to the curved (remaining) one, that is $\mathrm{S}=S_{1}+$ $\mathrm{S}_{2}$.

Using the model of Forrestal and Tzou (1997) and Ben-Dor et al. [3] the drag force $D$ of an axisymmetric striker of class $K_{1}$ of given length $L$ and final radius $R$ has two different expressions depending on the current penetration length $h$ :

$$
D=\left\{\begin{array}{l}
\eta h \text { if } 0 \leq h \leq 4 R \\
\int_{S} t_{z} d S \text { if } h \geq 4 R
\end{array}\right.
$$

where $\eta>0$ is an experimental constant; $d S$ is the elementary surface of the impactor and $t_{z}$ the component along $z$ of the normal stress $\sigma$ acting on $d S$ (see Figure 1). According to Forrestal and Tzou [5], $\sigma$ is equal to the following parabolic expression

$$
\sigma(v)=\sigma_{*}\left[A_{0}+A_{1} k v_{n}+A_{2}\left(k v_{n}\right)^{2}\right], k=\sqrt{\frac{\rho_{m}}{\sigma_{*}}}
$$

Where $\rho_{m}$ is the density of the shield, $\sigma_{*}$ its uniaxial compressive strength and $A_{0}, A_{1}, A_{2}$ are given positive dimensionless constants shown in Table 1 (see [5]):

The drag force $D$ when $h \geq 4 R$ (second stage of penetration), is caused by the force $D_{1}$ applied to the plane lateral surface $S_{1}$, and $D_{2}$, applied to the curved lateral surface $S_{2}$ of the impactor; so the total drag force $D$ is expressed by the following formula
Table 1. Coefficients for different models of the shield.

\begin{tabular}{ccccc}
\hline $\begin{array}{c}\text { Number of } \\
\text { the model }\end{array}$ & Characteristec of the model & $\mathrm{A}_{0}$ & $\mathrm{~A}_{1}$ & $\mathrm{~A}_{2}$ \\
\hline 1 & $\begin{array}{c}\text { Incompressible, } \\
\text { elastic-plastic }\end{array}$ & 5.18 & 0.00 & 3.88 \\
2 & $\begin{array}{c}\text { Incompressible, } \\
\text { elastic-cracked-plastic }\end{array}$ & 4.05 & 1.36 & 3.51 \\
3 & $\begin{array}{c}\text { Compressible, elastic-plastic } \\
4\end{array}$ & 4.50 & 0.75 & 1.29 \\
$\begin{array}{c}\text { Compressible, } \\
\text { elastic-cracked-plastic }\end{array}$ & 3.45 & 1.60 & 1.12 \\
\hline
\end{tabular}

$$
D=D_{1}+D_{2}
$$

Let us consider now a thin strip of impactor realized by cutting it with two planes distant one from the other $d z$ and orthogonal to $z$ axis (see Figure 1). The surface $d S$ between such planes is decomposed in two parts: $d S_{1}$ (plane part) shown in dark grey and $d S_{0}$ (curved part) showed in light grey. The elementary surface $d S_{1}$ has the following expression

$$
d S_{1}=2 d \frac{d z}{\sin \left(\alpha_{1}\right)}
$$

Where $\alpha_{1}$ is the angle between the negative direction of $z$ axis and the outer unit vector $\bar{n}_{1}$ normal to $S_{1}$ and $d$ is the segment shown in Figure 1(b) and (c). Taking into account that $\tan \left(\alpha_{1}\right)=R / L=p$ we can write

$$
d S_{1}=4 \sqrt{1+p^{2}} \sqrt{y^{2}-(p z)^{2}} d z
$$

Whereas from Figure 1 (b) and (c) we have

$$
d S_{2}=4 \overline{O P} \beta \sqrt{1+y^{\prime 2}} d z
$$


where prime denotes derivation with respect to $z$; moreover $\overline{O P}=y(z)$ and $\beta=\operatorname{asin}(p z / y)$ so

$$
d S_{2}=4 y \sqrt{1+y^{\prime 2}} \operatorname{asin}\left(\frac{p z}{y}\right) d z
$$

Remembering that only the normal component $t_{z}$ of $\sigma$ is responsible for the drag force, we can write

$$
D=D_{1}+D_{2}=\int_{S_{1}} t_{z} d S_{1}+\int_{S_{2}} t_{z} d S_{2}
$$

but

$$
t_{z}=\left\{\begin{array}{l}
\sigma \cos \left(\alpha_{1}\right)=\sigma \frac{p}{\sqrt{1+p^{2}}} \text { on } S_{1} \\
\sigma \cos \left(\alpha_{2}\right)=\sigma \frac{y^{\prime}}{\sqrt{1+y^{\prime 2}}} \text { on } S_{2}
\end{array}\right.
$$

so

$$
\begin{gathered}
D=\int_{S_{1}} t_{z} d S_{1}+\int_{S_{2}} t_{z} d S_{2}= \\
4 \int_{0}^{L} \sigma p \sqrt{y^{2}-(p z)^{2}} d z+4 \int_{0}^{L} \sigma y y^{\prime} \operatorname{asin}\left(\frac{p z}{y}\right) d z
\end{gathered}
$$

Substituting (2) in (8) we have

$$
\begin{gathered}
D=4 \sigma_{*} \int_{0}^{L}\left(A_{0}+A_{1} k v_{n}+A_{2}\left(k v_{n}\right)^{2}\right) p \sqrt{y^{2}-(p z)^{2}} d z+ \\
4 \sigma_{*} \int_{0}^{L}\left(A_{0}+A_{1} k v_{n}+A_{2}\left(k v_{n}\right)^{2}\right) y y^{\prime} \operatorname{asin}\left(\frac{p z}{y}\right) d z
\end{gathered}
$$

where $v_{n}$ has the expression

$$
v_{n}=\left\{\begin{array}{l}
v \cos \left(\alpha_{1}\right)=v \frac{p}{\sqrt{1+p^{2}}} \text { on } S_{1} \\
v \cos \left(\alpha_{2}\right)=v \frac{y^{\prime}}{\sqrt{1+y^{\prime 2}}} \text { on } S_{2}
\end{array}\right.
$$

Introducing the following dimensionless variables:

$$
\begin{gathered}
\tilde{D}=\frac{D}{\pi \sigma_{*} R^{2}}, \tilde{z}=\frac{z}{L}, \tilde{y}=\frac{y}{L} \\
\tilde{y}^{\prime}=y^{\prime}, \tau=\frac{R}{L}, V=k v
\end{gathered}
$$

(from now on tilde is omitted)

we get the following quadratic expression for $D$ :

$$
D=a_{0}+a_{1} V+a_{2} V^{2}
$$

where

$$
\begin{gathered}
a_{0}=A_{0}\left(B_{0} I_{1}+B_{1} I_{2}\right) \\
a_{1}=A_{1}\left(B_{0} B_{2} I_{1}+B_{1} I_{3}\right) \\
a_{2}=A_{2}\left(B_{0} B_{2}^{2} I_{1}+B_{1} I_{4}\right)
\end{gathered}
$$

$$
\begin{gathered}
B_{0}=\frac{4 p}{\pi \tau^{2}} \\
B_{1}=\frac{4}{\pi \tau^{2}} \\
B_{2}=\frac{p}{\sqrt{1+p_{2}}} \\
I_{1}=\int_{0}^{1} \sqrt{y^{2}-(p z)^{2}} d z \\
I_{2}=\int_{0}^{1} \beta y y^{\prime} d z \\
I_{3}=\int_{0}^{1} \beta \frac{y y^{\prime 2}}{\sqrt{1+y^{\prime 2}}} d z \\
I_{4}=\int_{0}^{1} \beta \frac{y y^{\prime 3}}{1+y^{\prime 2}} d z
\end{gathered}
$$

Now applying the equation of motion of the impactor (for details see [3] we find the following expression

$$
P=1+\omega \int_{0}^{V^{*}} \frac{V}{a_{0}+a_{1} V+a_{2} V^{2}} d V
$$

where

$$
\begin{aligned}
& P=\frac{D O P}{4 R} \text { is the dimensionless expression of DOP } \\
& \omega=\frac{m}{4 R^{3} \pi k} \text { is an dimensionless coefficient }
\end{aligned}
$$

$V^{*}$ is the adimensional velocity of the impactor for $h=4 R$ found imposing that for $h=4 R$ the expression of the drag force $D$ in the first stage of penetration has the same value of $D$ in the second stage of penetration [3]:

$$
V^{*}=\frac{-a_{1}+\sqrt{a_{1}^{2}-4\left(a_{0}-\omega\right) W^{2}}\left(a_{2}+\omega\right)}{2\left(a_{2}+\omega\right)}
$$

where $W=k v_{i m p}$ is the dimensionless impact velocity. Of course to get real values of $V^{*}$ the discriminant in (17) must be nonnegative.

\section{Building a Better Solution}

In the case of shape of revolution of class $K_{0}$ Ben-Dor et $a l$. [3] found the solution for a large set of problem parameters and showed that the optimal shape has a flat nose and is concave. Let us now show with an example that using a shape of class $K_{1}$ a better solution than that corresponding to class $K_{0}$ is obtained. To begin, we consider the following problem parameters: model number: 3(compressible elastic-plastic shield); $\omega=14 ; \tau=0.5$; $W=3.5$. The optimum DOP value for a body of class $K_{0}$ is $P=14.1$ [3]. Using the same problem parameters, let 
us now build a shape of class $K_{1}$ choosing a generatrix of the form

$$
y(z)=a z^{b}+c
$$

where $a=0.491 ; b=0.38 ; c=5.616 \times 10^{-3} \quad$ (Figure 2).

Now, applying Equation (13)-(17) we found that $P=$ $14.4>14.1$. This is sufficient to show that class $K_{1}$ can give better solutions than class $K_{0}$. We note that several theoretical and numerical considerations about screwdriver shapes can be found in [14] and the references given there, although applied to the Newton problem of optimal aerodynamic bodies.

\section{Formulation of the Optimum Design}

Our task is not only to show that class $K_{1}$ can give better solutions than class $K_{0}$ as we have seen in the previous section, but also to find the generatrix $y(z)$ for which the corresponding impactor shape belonging to $K_{1}$ maximizes DOP:

$$
\begin{gathered}
D O P=D O P(y) \rightarrow \max _{y} \\
\text { with } y(0)=y_{0}(\text { free }) \text { and } y(1)=\tau(\text { fixed })
\end{gathered}
$$

We shall show in the following part of the paper one possible strategy of solution.

\section{Psoa Algorithm}

To solve this problem we will use the method PSOA (Particle Swarm Optimization Algorithm), a recent heuristic suitable for finding global optima solutions. PSOA was introduced about one decade ago $[15,16]$, inspired by the behaviour of school of fish, flocks of birds or swarm of bees observed in nature. Like genetic algorithms (GA) and ant colony optimization methods (ACO), PSOA is also a stochastic, population-based global search algorithm. In a natural swarm each individual or particle changes position toward better places, to reach food or to escape from predators, exchanging information with the neighbourhood and without any central control. In the mathematical model at each individual corresponds a particle $i$ and at every particle $i$ is associated a position vector $\bar{x}_{i}$ in a $n$-dimensional space, to which corresponds a certain value of the objective function $f\left(\bar{x}_{i}\right)$ that represents the quality of $\bar{x}_{i}$; of course $\bar{x}_{i}$ should be a feasible solution to the problem under study. If some constraints must be considered, the objective function can be modified using the penalty technique. When the algorithm starts, a collection (swarm) of $m$ particles is randomly chosen using a uniform probability distribution. Then the vector position of each particle is updated at every iteration adding a displace

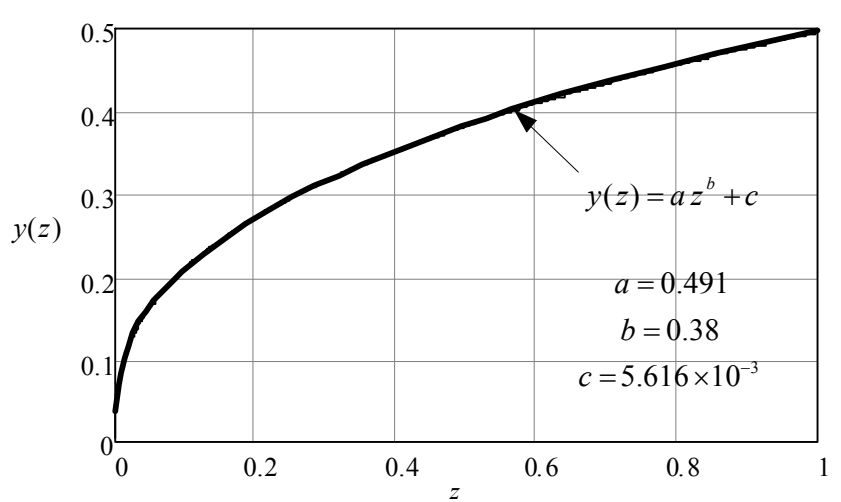

Figure 2. A solution on class $K_{1}$.

ment based on the information about the previous swarm positions; the knowledge of the gradient of $f$ is not needed. So the iteration rule is the following

$$
\bar{x}_{i}^{(k+1)}=\bar{x}_{i}^{(k)}+\bar{S}_{i}^{(k+1)}
$$

Where $\bar{x}_{i}^{(k)}$ and $\bar{x}_{i}^{(k+1)}=$ positions of particle $i$ at $k$-th and $k+1$-iteration, $\bar{s}_{i}^{(k+1)}=$ displacement of particle $i$ at $k+1$-iteration. The formula for ${\overline{S_{i}}}^{(k+1)}$ is

$$
\bar{s}_{i}^{(k+1)}=w \bar{s}_{i}^{(k)}+c_{1} r_{1}\left(\bar{p}_{i}-\bar{s}_{i}^{(k)}\right)+c_{2} r_{2}\left(\bar{p}_{g}-\bar{s}_{i}^{(k)}\right)
$$

Formula (21) is the core of the algorithm and contains some fundamental information about the neighbourhood of particle $i: w$ is called inertia and serves to control the influence of the previous displacement on the new one. Recommended values of $w$ range from 0 to $1.4 ; \bar{p}_{i}$ is the best position found for the particle $i$ to iteration $k$, whereas $\bar{p}_{g}$ is the best position inside the swarm up to iteration $k ; c_{1}$ and $c_{2}$ are two positive constants used to balance the cognitive and social aspect: usually $c_{1}=c_{2}=2$. $r_{1}$ and $r_{2}$ are random parameters in the range [0,1], sampled from a uniform distribution. The algorithm stops when a prescribed number of consecutive iterations without improvement in the objective function is reached. From (20) and (21) it is clear that PSOA takes account of the previous random walks of particles. During iterations typically two main problems can arise: the first is the explosion of the swarm: it means that the maximum distance between two particles grows indefinitely and consequently an overflow error occurs; the second is the stagnation of the swarm which means that all the particles occupy the same position, leading to a suboptimalsolution. To try to avoid such inconveniences the two following additional rules are included in the algorithm: the first rule is the limitation of the maximum displacement: $\left|\bar{s}_{i}^{(k)}\right| \leq s_{\max }$ where $s_{\max }$ is a constant which is 
problem dependent; the second one is called craziness: with an assigned probability $P_{c r}$ (for example 0.005 ), the displacement $\bar{s}_{i}^{(k+1)}$ is not calculated with (21) but completely randomly, with the only condition that its modulus must be $\leq s_{\max }$. The PSOA algorithm preposed has several advantages with respect to classical optimization algorithms: 1) it is able to find the true optimum solution in the entire search space (global optimum); 2) it is easy to implement; 3 ) it can handle non-differentiable function and no calculation of derivatives is required. The main disadvantage is that it can be quite timeconsuming. The PSOA algorithm so far described is only one among many others possible that can be found in the current literature [15,17-19]. For the problem under consideration the swarm is formed by $m$ particles and each particle is a generatrix $y_{i}(z)$ to which corresponds an impactor shape of class $K_{1}$ and a certain value of the objective function $f$ (DOP); each generatrix is approximated by the vector $\overline{y_{i}}$ where every component of $\overline{y_{i}}$ is a value of $y_{i}$; the $\mathrm{z}$ axis has been discretized into $n$ - 1 intervals so we have $n$ points in which to calculate the generatrix; as a consequence the vector $y_{i}$ belongs to an $n$-dimensional space; because we know in advance that the most critical zone of the impactor shape in near $z=$ 0 , the $n-1$ intervals of $\mathrm{z}$ axis are not equals, but shorter near $z=0$ and longer as we go toward $z=1$. In our calculations $n$ (which corresponds to the swarm size) has been fixed to 30. In Figure 3 some generatrices of optimum impactors of class $K_{1}$ are shown using the following common parameters: Model n. $3, \tau=0.5, \omega=14$ for different impactor's speed $\mathrm{W}=1.0,2.0,3.0,3.5$.

In Table 2 the comparison of DOP of impactors of class $K_{1}$ illustrated in Figure 3, and the corresponding impactors of class $K_{0}$ (surfaces of revolution) with the same problem parameters model $n .3, \tau=0.5, \omega=14$, is shown for $\mathrm{W}=1.0,2.0,3.0,3.5$.

Table 2 shows clearly that the classical and intuitive search space of axisymmetric high speed impactors does not guarantee the best solution (i.e. the maximum DOP). In fact in the cases studied show that an impactor shape belonging to a more general class $K_{1}$ (non-axisymmetric shape called "screwdriver shape"), can give better results.

\section{Concluding Remarks}

Starting from the mechanical model proposed by Forrestal et al. and using formulas obtained by Ben-Dor et al. for finding the best impactor shape in the class $K_{0}$ of axisymmetric bodies, we presented a different shape of impactors in the new class $K_{1}$ which is different from the standard axisymmetric shape, and show that bodies belonging to class $K_{1}$ can give better results compared to corresponding $K_{0}$ bodies. We also finalized a code based

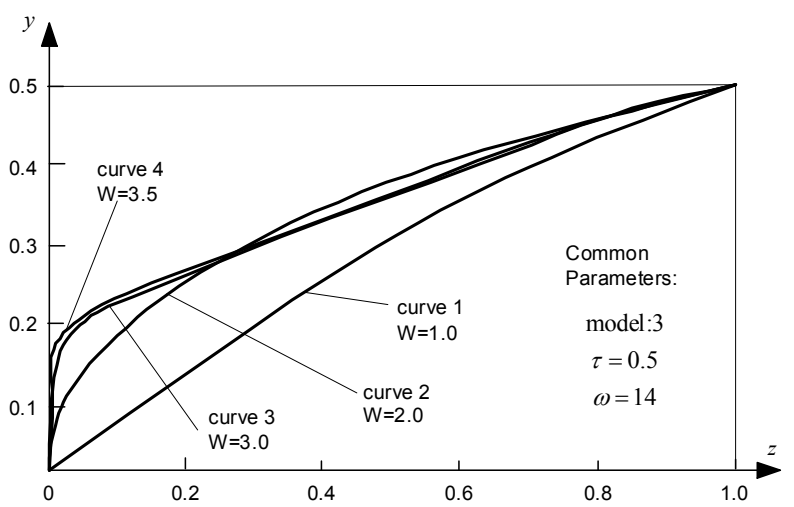

Figure 3. Some generatrices of optimum impactors.

Table 2. Comparison of DOP of impactors.

\begin{tabular}{|c|c|c|c|}
\hline \multicolumn{2}{|c|}{$\begin{array}{c}\text { DOP of impactors of class class } \\
\mathrm{K}_{1} \text { with the following common } \\
\text { parameters: } \\
\text { modeln.3, } \tau=0.5, \omega=14\end{array}$} & \multicolumn{2}{|c|}{$\begin{array}{c}\text { Corresponding DOP of } \\
\text { impactors of class } \mathrm{K}_{0} \text { using } \\
\text { the same parameters: } \\
\text { modeln.3, } \tau=0.5, \omega=14\end{array}$} \\
\hline $\begin{array}{c}\text { Impaactor's } \\
\text { speed }\end{array}$ & $\begin{array}{c}\text { DOP of impactor } \\
\text { of class } \mathrm{K}_{1}\end{array}$ & $\begin{array}{c}\text { Impactor's } \\
\text { speed }\end{array}$ & $\begin{array}{c}\text { DOP of } \\
\text { impactor of } \\
\text { class } \mathrm{K}_{0}\end{array}$ \\
\hline $\begin{array}{c}\mathrm{W}=1.0 \\
\text { curve } 1 \text { on } \\
\text { figure.3) }\end{array}$ & 2.0 & $\mathrm{~W}=1.0$ & 2.0 \\
\hline $\begin{array}{c}\mathrm{W}=2.0 \\
\text { (curve } 2 \text { on } \\
\text { figure.3) }\end{array}$ & 6.0 & $\mathrm{~W}=2.0$ & 5.8 \\
\hline $\begin{array}{c}\mathrm{W}=3.0 \\
\text { (curve } 3 \text { on } \\
\text { figure.3) }\end{array}$ & 11.6 & $\mathrm{~W}=3.0$ & 11.2 \\
\hline $\begin{array}{c}\mathrm{W}=3.5 \\
\text { (curve } 4 \text { on } \\
\text { figure.3) }\end{array}$ & 14.7 & $\mathrm{~W}=3.5$ & 14.1 \\
\hline
\end{tabular}

on PSOA heuristic algorithm to find the best generatrix in class $K_{0}$ once some problem parameters are fixed. Some results are also given in a tabular form. We must emphasize that the class $K_{1}$ is not the most general class of impactors we can imagine so the problem of the best impactor remains open.

\section{References}

[1] G. Ben-Dor, A. Dubinsky and T. Elperin, "Numerical Solutions for Shape Optimization of an Impactor Penetrating into a Semi-infinite Target," Computers and Structures, Vol. 81, No. 1, 2003, pp. 9-14.

[2] G. Ben-Dor, A. Dubinsky, T. Elperin, "Shape Optimization of an Impactor Penetrating into a Concrete or a Limestone Target," International Journal of Solids and Structures, Vol. 40, No. 17, 2003, pp. 4487-4500.

[3] G. Ben-Dor, A. Dubinsky and T. Elperin, "Modeling of High-speed Penetration into Concrete Shields and Shape Optimization of Impactors," Mechanics Based Design of Structures and Machines, Vol. 34, No. 2, 2006, pp. 139-156.

[4] N. V. Banichuk, V. M. Petrov and F. L. Chernousko, "The Method of Local Variations for Variational Prob- 
lems Involving Non-additive Functionals," USSR Computational Mathematics and Mathematical Physics, Vol. 9, No. 3, 1969, pp. 66-76.

[5] M. J. Forrestal and D. Y. Tzou, "A Spherical Cavity-expansion Penetration Model for Concrete Targets," International Journal of Solids and Structures, Vol. 34, No. 31-32, 1997, pp. 4127-4146.

[6] M. J. Forrestal, B. S. Altman, J. D. Cargile and S. J. Hanchak, "An Empirical Equation for Penetration Depth of Ogive-nose Projectiles into Concrete Targets," International Journal of Impact Engineering, Vol. 15, No. 4, 1994, pp. 396-405.

[7] M. J. Forrestal, D. J. Frew, S. J. Hanchak and S. Brar, "Penetration of Grout and Concrete Targets with Ogivenose Steel Projectiles," International Journal of Impact Engineering, Vol. 18, No. 5, 1996, pp. 465-476.

[8] G. Ben-Dor, A. Dubinsky and T. Elperin, "Applied Highspeed Plate Penetration Dynamics," Springer, Berlin, 2006.

[9] A. I. Bunimovich and G. E. Yakunina, "On the Shape of Minimum-resistance Solids of Revolution Moving in Plastically Compressible and Elastic-Plastic Media,"_Journal of Applied Mathematics and Mechanics, Vol. 51, No. 2 , 1987, pp. 386-392.

[10] N. A. Ostapenko and G. E. Yakunina, "The Shape of Slender Three Dimensional Bodies with Maximum Depth of Penetration into Dense Media," Journal of Applied Mathematics and Mechanics, Vol. 63, 1999, pp. 953-967.

[11] G. E. Yakunina, "On Body Shapes Providing Maximum Penetration Depth in Dense Media," Doklady Physics,
Vol. 46, No. 2, 2001, pp. 140-143.

[12] G. E. Yakunina, "On the Optimal Shapes of Bodies Moving in Dense Media," Doklady Physics, Vol. 50, No. 12, 2005, pp. 650-654.

[13] N. V. Banichuk and S. Y. Ivanova, "Shape Optimization of Rigid 3-D High-speed Impactor Penetrating into Concrete Shields," Mechanics Based Design of Structures and Machines, Vol. 36, 2008, pp. 249-259.

[14] D. Bucur and G. Buttazzo, "Variational Methods in Shape Optimization Problems," Birkhauser, Boston, 2005.

[15] R. C. Eberhart and J. Kennedy, "A New Optimizer Using Particle Swarm Theory," Proceedings of the 6th International Symposium on Micromachine and Human Science, Nagoya, 1995, pp. 39-43.

[16] J. Kennedy and R. C. Eberhart, "Particle Swarm Optimization," Proceedings of the IEEE International Joint Conference on Neural Networks, Perth, 1995, pp. 1942-1948.

[17] M. Clerc, "Particle swarm optimization," ISTE, London, 2006.

[18] M. M. Ali and P. Kaelo, "Improved particle swarm algorithms for global optimization," Applied Mathematics and Computation, Vol. 196, 2008, pp. 578-593.

[19] P. C. Fourie and A. A. Groenwold, "The Particle Swarm Optimization Algorithm in Size and Shape Optimization," Structural and Multidisciplinary Optimization, Vol. 23, No. 4, 2002, pp. 259-267.

[20] A. P. Engelbrecht, "Fundamentals of computational swarm intelligence," Wiley, Chichester, 2005 